\title{
The Eruption of a Small-scale Emerging Flux Rope as the Driver of an M-class Flare and of a Coronal Mass Ejection
}

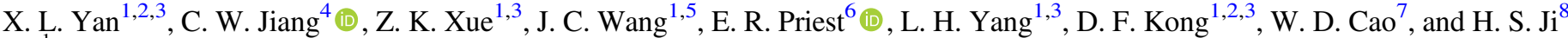 \\ ${ }^{1}$ Yunnan Observatories, Chinese Academy of Sciences, 396 Yangfangwang, Guandu District, Kunming 650216, Yunnan, P. R. China; yanxl@ynao.ac.cn \\ ${ }^{2}$ Key Laboratory of Solar Activity, National Astronomical Observatories, Chinese Academy of Sciences, Beijing 100012, P. R. China \\ ${ }^{3}$ Center for Astronomical Mega-Science, Chinese Academy of Sciences, 20A Datun Road, Chaoyang District, Beijing, 100012, P. R. China \\ ${ }^{4}$ Institute of Space Science and Applied Technology, Harbin Institute of Technology, Shenzhen, 5180055, P. R. China \\ ${ }^{5}$ Graduate School of University of Chinese Academy of Sciences, Yuquan Road, Shijing-shang Block Beijing 100049, P. R. China \\ ${ }^{6}$ Mathematics Institute, University of St Andrews, St Andrews, KY16 9SS, UK \\ ${ }^{7}$ Big Bear Solar Observatory, 40386 North Shore Lane, Big Bear City, CA 92314, USA \\ ${ }^{8}$ Key Laboratory for Dark Matter and Space Science, Purple Mountain Observatory, Chinese Academy of Sciences, Nanjing 210008, Jiangsu, P. R. China \\ Received 2017 May 3; revised 2017 June 22; accepted 2017 June 23; published 2017 August 8
}

\begin{abstract}
Solar flares and coronal mass ejections are the most powerful explosions in the Sun. They are major sources of potentially destructive space weather conditions. However, the possible causes of their initiation remain controversial. Using high-resolution data observed by the New Solar Telescope of Big Bear Solar Observaotry, supplemented by Solar Dynamics Observatory observations, we present unusual observations of a small-scale emerging flux rope near a large sunspot, whose eruption produced an M-class flare and a coronal mass ejection. The presence of the small-scale flux rope was indicated by static nonlinear force-free field extrapolation as well as data-driven magnetohydrodynamics modeling of the dynamic evolution of the coronal three-dimensional magnetic field. During the emergence of the flux rope, rotation of satellite sunspots at the footpoints of the flux rope was observed. Meanwhile, the Lorentz force, magnetic energy, vertical current, and transverse fields were increasing during this phase. The free energy from the magnetic flux emergence and twisting magnetic fields is sufficient to power the M-class flare. These observations present, for the first time, the complete process, from the emergence of the small-scale flux rope, to the production of solar eruptions.
\end{abstract}

Key words: Sun: activity - Sun: coronal mass ejections (CMEs) - Sun: flares - Sun: magnetic fields - Sun: photosphere - sunspots

Supporting material: animations

\section{Introduction}

Solar flares and coronal mass ejections (CMEs) are explosive phenomena observed in the solar atmosphere (Wang et al. 2002). A huge amount of free magnetic energy stored in the solar atmosphere is released during their eruptions (Forbes 2000; Priest \& Forbes 2002; Schmieder et al. 2015). This energy is transformed into radiative energy, bulk kinetic energy, as well as thermal and nonthermal energy (Deng et al. 2013; Lin et al. 2015; Xiang \& Qu 2016).

Previous observations have demonstrated that the accumulation of free magnetic energy in the corona may be due to shearing motion (Wang et al. 1994; Moore et al. 2012), sunspot rotation (Régnier \& Canfield 2006; Yan \& Qu 2007; Zhang et al.

2007; Yan et al. 2009; Sturrock \& Hood 2016; Zheng et al. 2017), and magnetic emergence or cancellation (Wang \& Shi 1993; Chen \& Shibata 2000; Sterling et al. 2010) in the photosphere. However, the detailed processes for solar eruptions have been unclear for many years because it is difficult to deduce the three-dimensional magnetic structure in the corona. Several possible models have been proposed for eruptions of flux ropes and the associated reconnection, namely, magnetic breakout, tether-cutting, magnetic non-equilibrium, kink instability, and torus instability (Carmichael 1964; Sturrock 1966; Hirayama 1974; Kopp \& Pneuman 1976; Shibata et al. 1995; Antiochos et al. 1999; Lin \& Forbes 2000; Moore et al. 2001; Amari et al. 2003; Török et al. 2004; Kliem \& Török 2006; Schrijver et al. 2008; Shen et al. 2012; Kliem et al. 2014; Chen et al. 2016; Shen et al. 2017).
With the improvement of the observations, more and more fine structures of the Sun are revealed during solar eruptions. The model of the flux rope in solar eruptions has been proposed for many years (van Ballegooijen \& Martens 1989; Rust \& Kumar 1994, 1996). Until recent years, the existence of flux ropes in the solar atmosphere has been evidenced using highresolution observational data (Tian et al. 2010; Cheng \& Ding 2016; Hou et al. 2016; Li et al. 2016a, 2016b; Lim et al. 2016; Zhou et al. 2016). Zhang et al. (2012) reported that a flux rope with a twisted and writhed sigmoidal structure observed by the Solar Dynamics Observatory (SDO) manifests itself as a hot channel before and during the solar eruption. A similar observation of a magnetic cloud as the counterpart of a hot channel was studied by Song et al. (2015). They found that the hot channel corresponds to a flux rope in the solar corona. The existence of flux ropes can also be tracked out by material from a surge in a failed filament eruption (Yang et al. 2014). Using New Solar Telescope (NST) observations, Wang et al. (2015) also found that a set of loops developed into a flux rope before a two-ribbon flare. Filippov et al. (2015) presented several filaments with highly twisted magnetic structure observed by the Transition Region And Coronal Explorer (TRACE) and Big Bear Solar Observatory (BBSO). These observations reveal that flux ropes may be ubiquitous in the solar atmosphere (Zhang et al. 2015a).

However, it is still an open question how flux ropes form. Some models proposed that the photospheric activities can result in the formation of flux ropes via sunspot rotation, shearing 
motion, convergence flow, magnetic reconnection, and magnetic cancellation (van Ballegooijen \& Martens 1989; Moore et al. 2001; Fan 2009; Priest \& Longcope 2017). Recently, more and more observational evidence of flux rope formation has been obtained from high-resolution observations. Yan et al. (2012, 2015), Yang et al. (2015), and James et al. (2017) found that the formation of the S-shaped filaments or flux ropes is closely related to rotating sunspots that one foot of the filaments is rooted in. This scenario is confirmed by the research of Vemareddy et al. (2016). They found that the magnetic connections of the sigmoid are driven by the slow motion of sunspot rotation, which finally transforms into a highly twisted flux rope structure. Joshi et al. (2014) presented a clear case of the formation of a compound flux rope via the merging of two nearby filament channels. Song et al. (2014) presented a direct observation of the flux rope formation process from a loop arcade during an eruption, which was associated with an M-class flare and a coronal mass ejection (CME) that occurred at the southwest limb on 2013 November 21. Yan et al. (2016) reported that the formation of an inverse S-shaped flux rope was due to the reconnection of two groups of chromospheric fibrils from observations made with the $1 \mathrm{~m}$ New Vacuum Solar Telescope (NVST) (Liu et al. 2014). Kumar et al. (2017) found that the reconnection between the cool $\mathrm{H} \alpha$ loops in the chromosphere during the flares can form an unstable flux rope using highresolution observations from the $1.6 \mathrm{~m}$ New Solar Telescope (NST) at BBSO. Some indirect evidence of the existence of flux ropes can be deduced by using a nonlinear force-free field (NLFFF) extrapolation (Cheng et al. 2010; Jiang et al. 2014, 2016a, 2016b).

A model of the emergence of a flux rope has also been proposed before solar eruptions by some researchers (Rust \& Kumar 1994; Fan 2009). Observational evidence is very rare, however. Up to now, only one case was reported of the emergence of a flux rope, and this was by Okamoto et al. (2009). They found that a helical flux rope emerged from below the photosphere into the corona along the polarity inversion line (PIL) below the preexisting prominence according to the observation that the orientations of the horizontal magnetic fields along the PIL in the photosphere gradually changed with time from a normal-polarity configuration to an inverse-polarity configuration, and the horizontal magnetic field region exhibited blueshift. However, Vargas Dominguez et al. (2012) argued that the signatures presented by Okamoto et al. (2009) were not sufficient indicators of a twisted flux tube emergence. They found a decrease in the unsigned flux at the PIL rather than the expected increase in the case studied by Okamoto et al. (2009). Furthermore, no shear motion and converging flows were detected in Okamoto's research. Several simulations were carried out to address the emergence of convection zone flux tubes. For instance, Archontis \& Hood (2013) presented three-dimensional magnetohydrodynamics (MHD) simulations of the formation of jets triggered by the emergence and eruption of solar magnetic fields. The similar triggering process of jet and surge were obtained by simulations of Moreno-Insertis \& Galsgaard (2013) and Nóbrega-Siverio et al. (2016). Leake et al. (2014) performed three-dimensional MHD simulations of the emergence of flux tubes from the convection zone into a preexisting dipole coronal field. They found that the external reconnection between the emergence flux tube and the overlying magnetic field lines is vital to the eruption process. That is to say, this external reconnection helps the further expansion of the coronal flux rope into the corona. MacTaggart \& Haynes (2014) considered the formation of two flux ropes in an MHD solar flux emergence simulation. In their simulation, a shearing motion along the PIL was found during the formation of two flux ropes. Moreover, when the first rope approached the top boundary of the domain, it was dissipated. The second flux rope emerged as a result of the decrease in overlying coronal magnetic fields by reconnection between the emerging flux region and the ambient magnetic fields.

Here we use NST and SDO mutiwavelength data to investigate the process of the emergence of a small-scale flux rope to the production of an M-class flare and a small CME. Observations and methods are presented in Section 2. The results are shown in Section 3. Conclusions and discussions are given in Section 4.

\section{Observations and Methods}

\subsection{Observations}

TiO (7057 $\AA$ ) images and H-alpha images are obtained from the Visible Imaging Spectrometer (VIS) on the $1.6 \mathrm{~m}$ New Solar Telescope at BBSO (Cao et al. 2010; Goode \& Cao 2010). TiO images have a spatial resolution of 0. " 0342 per pixel and the time cadence is $15 \mathrm{~s}$. H-alpha images have a pixel size is 0 ". 0295 , and the cadence is $43 \mathrm{~s}$ for VIS to complete a scan at a $0.2 \AA$ step from $-1.0 \AA$ to $+1.0 \AA$ around the $\mathrm{H}$-alpha line center. $\mathrm{H}$-alpha blue-wing $(-0.6 \AA), \mathrm{H}$-alpha center, and $\mathrm{H}$-alpha red-wing $(+0.6 \AA)$ images are used in this study.

Full-disk UV and EUV images with a $12 \mathrm{~s}$ cadence and a spatial resolution of 0 " 6 per pixel observed by $S D O /$ AIA (Lemen et al. 2012) are employed to show the process of flux rope eruption. The vector magnetograms from Space Weather HMI Active Region Patch (SHARP) series observed by the Helioseismic and Magnetic Imager (HMI) (Schou et al. 2012; Bobra et al. 2014; Centeno et al. 2014) have a pixel scale of about 0 ". 5 and a cadence of 12 minutes. They are derived using the Very Fast Inversion of the Stokes Vector algorithm (Borrero et al. 2011). The minimum energy method (Metcalf 1994; Metcalf et al. 2006; Leka et al. 2009) is used to resolve the 180 degree azimuthal ambiguity. The images are remapped using Lambert (cylindrical equal area) projection centered on the midpoint of the active region (AR), which is tracked at the Carrington rotation rate (Sun 2013).

\subsection{Methods}

\subsubsection{Current Calculation}

The calculation of current is based on Ampère's law:

$$
\boldsymbol{J}=\frac{c}{4 \pi}(\nabla \times \boldsymbol{B})
$$

in which $\mu_{0}$ is the vacuum magnetic permeability and $\boldsymbol{B}$ denotes the vector magnetic fields. The current density perpendicular to the solar surface can be calculated from Ampère's law by using HMI vector magnetograms according to the equation

$$
j_{z}=\frac{c}{4 \pi}(\nabla \times \boldsymbol{B})_{z}=\frac{c}{4 \pi}\left(\frac{\partial B_{x}}{\partial y}-\frac{\partial B_{y}}{\partial x}\right),
$$

where $B_{x}$ and $B_{y}$ are the two components of the photospheric horizontal magnetic field. The distribution of $j_{z}$ on the solar surface is obtained every 12 minutes. Note that all the equations in this paper are written in cgs. 

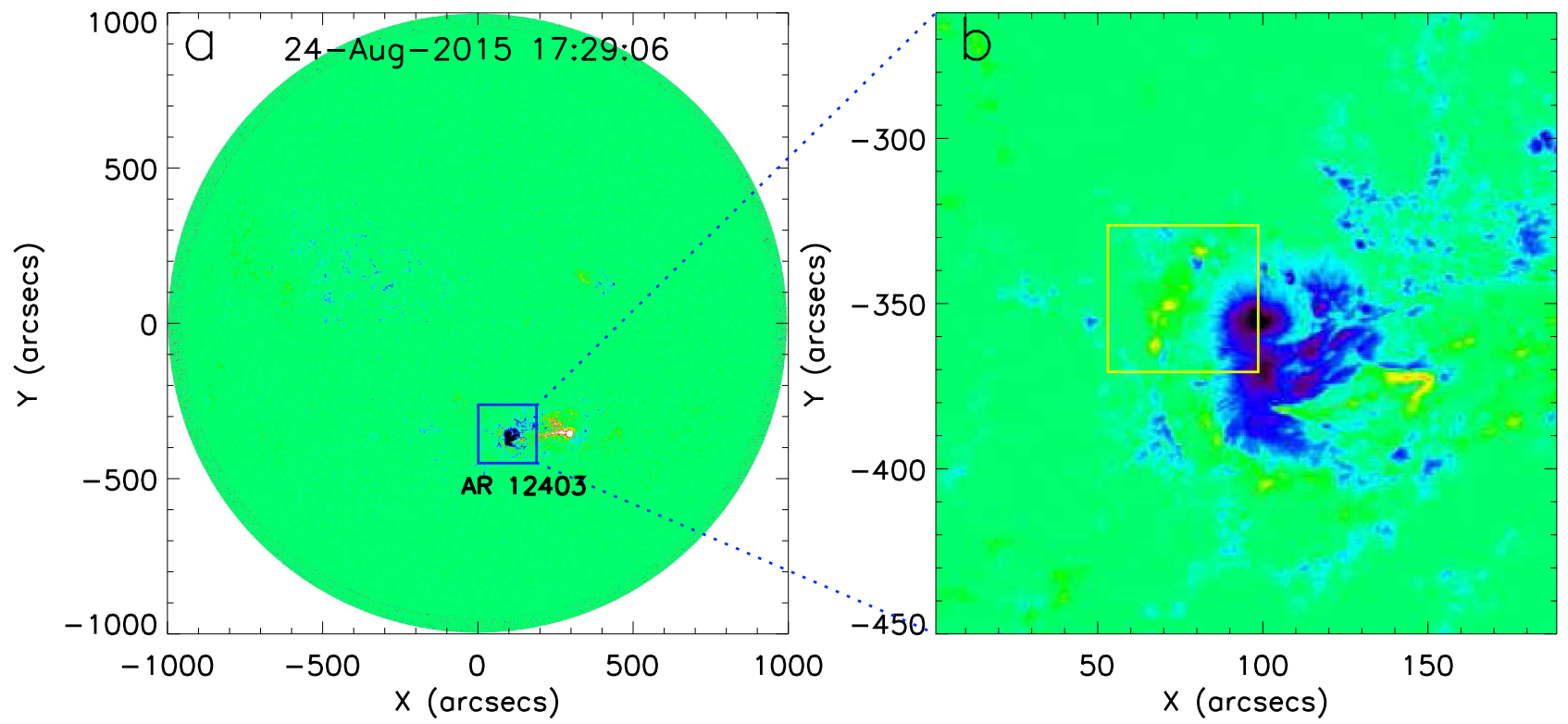

Figure 1. Left panel: full-disk line-of-sight magnetogram showing active region NOAA 12403 on the solar disk. Right panel: the following sunspots of the active region. The yellow box outlines the field of view of Figures 2 and 5.

\subsubsection{Calculation of the Magnetic Flux}

The integrated positive $\left(\phi_{\mathrm{zp}}\right)$ and negative $\left(\phi_{\mathrm{zn}}\right)$ magnetic fluxes are calculated from

$$
\begin{aligned}
\phi_{\mathrm{zp}} & =\int B_{z+} d A \\
\phi_{\mathrm{zn}} & =\int\left|B_{z-}\right| d A,
\end{aligned}
$$

where $B_{z+}$ and $B_{z-}$ are the vertical positive and negative magnetic fields, and $d A$ denotes the area differential.

\subsubsection{NLFFF Extrapolation}

The vector magnetic fields obtained by $S D O / \mathrm{HMI}$ are taken as the boundary condition to extrapolate the magnetic fields from the photosphere to the corona. The optimization algorithm proposed by Wheatland et al. (2000) and implemented by Wiegelmann (2004) is used to extrapolate the three-dimensional NLFFF structure of the active region. A preprocessing procedure (fff_temp_pre.pro in SSW) is used to smoothen the bottom boundary vector data before the extrapolation. This removes most of the net force and torque that would result in an inconsistency between the forced photospheric magnetic field and the force-free assumption in the NLFFF models (Wiegelmann et al. 2006).

\subsubsection{Lorentz Force Calculation}

The Lorentz force at the photospheric surface is divided into radial and horizontal components, which can be written as follows:

$$
\begin{gathered}
F_{\mathrm{r}}=\frac{1}{8 \pi} \int_{A_{p h}}\left(B_{h}{ }^{2}-B_{r}{ }^{2}\right) d A \\
F_{\mathrm{h}}=\frac{1}{8 \pi} \int_{A_{p h}} B_{h} B_{r} d A .
\end{gathered}
$$

Here, $B_{h}$ and $B_{r}$ represent the horizontal and radial components of $B$, which are parallel and vertical to the photosphere, respectively.
$A_{p h}$ is the area of the white box in the photosphere. These results are derived from the integral of the divergence of the Maxwell stress tensor.

The total Lorentz force acting on plasma at and above the solar photosphere can be written in terms of surface integrals of products of the photosphere field components $\boldsymbol{B}=\left(B_{x}, B_{y}, B_{y}\right)$ by assuming that the contributions from the top and side boundaries are negligible (Fisher et al. 2012). Thus, the horizontal $\left(F_{x}, F_{y}\right)$ and vertical $\left(F_{z}\right)$ components can be written as follows:

$$
\begin{gathered}
F_{\mathrm{x}}=\frac{1}{4 \pi} \int_{A_{p h}} B_{x} B_{z} d A \\
F_{\mathrm{y}}=\frac{1}{4 \pi} \int_{A_{p h}} B_{y} B_{z} d A \\
F_{\mathrm{z}}=\frac{1}{8 \pi} \int_{A_{p h}}\left(B_{x}{ }^{2}+B_{y}{ }^{2}-B_{z}{ }^{2}\right) d A .
\end{gathered}
$$

\subsubsection{Free Energy Calculation}

We use the NLFFF extrapolation to obtain the magnetic fields above the photosphere. Then the extrapolated NLFFF fields $\left(B_{\text {ext }}\right)$ and potential fields $\left(B_{\text {pot }}\right)$ are used to calculate the free energy from the equation

$$
E_{\text {free }}=\frac{1}{8 \pi} \int_{v}\left(B_{\mathrm{ext}}^{2}-B_{\mathrm{pot}^{2}}{ }^{2} d V,\right.
$$

where $B_{\text {ext }}$ and $B_{\text {pot }}$ indicate the extrapolated NLFFF fields and potential fields, respectively. $\mathrm{V}$ is the cube with a height of ten arcseconds, whose bottom is marked by the box in Figure 3(b).

\subsubsection{Data-driven Simulation}

Vector magnetograms from Space Weather HMI Active Region Patch (SHARP) series with a pixel scale of about 0.15 and a cadence of 12 minutes were used to derive the MHD model. The images were remapped using a Lambert (cylindrical equal area) projection centered on the midpoint of the AR, 


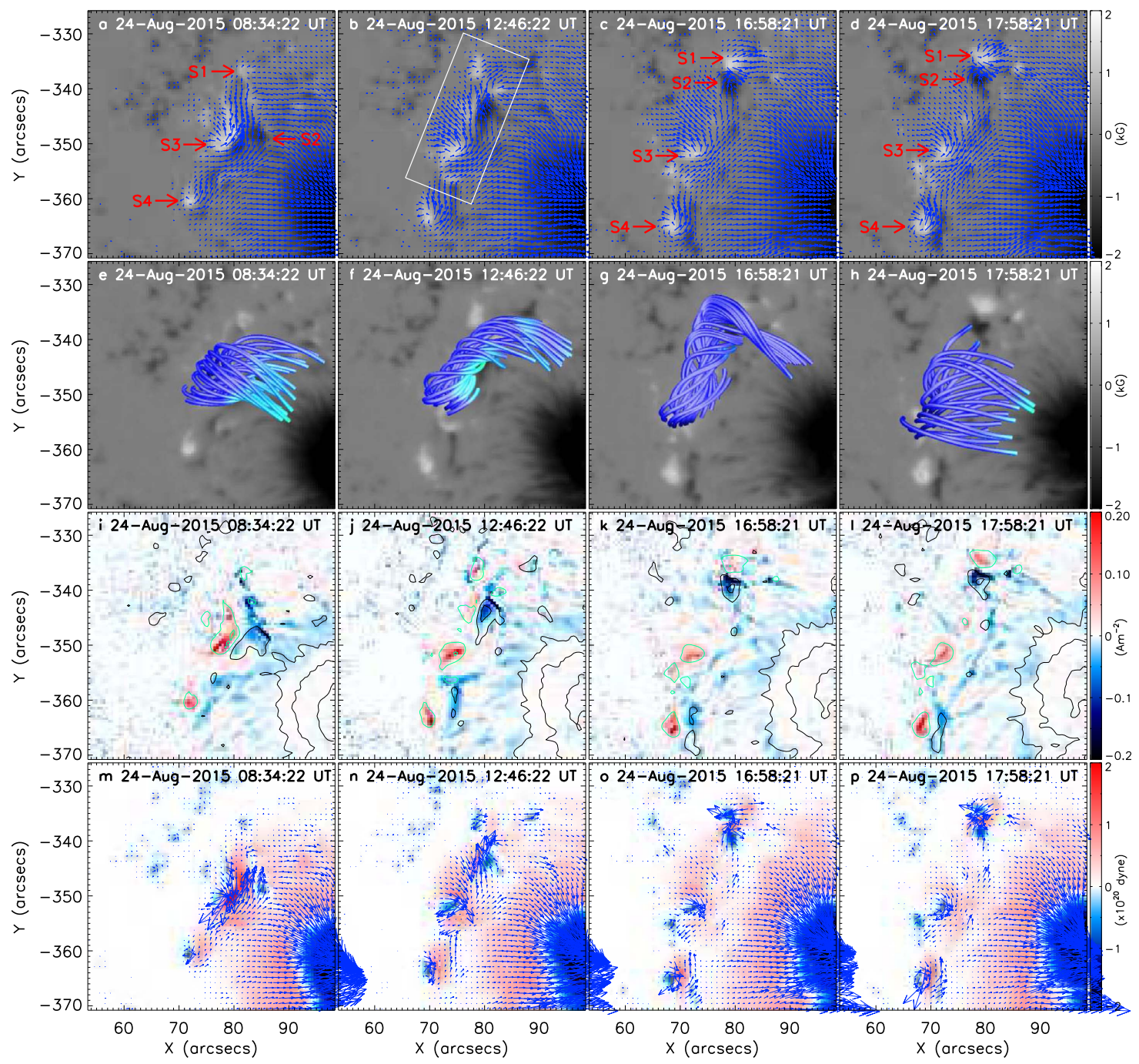

Figure 2. Evolution of the vector magnetograms, the magnetic structure of the NLFFF extrapolation, the electric current, and the Lorentz force. (a)-(d) Vector magnetograms observed by SDO/HMI. The red arrows indicate the small satellite sunspots. The white box shows the region used to calculate the magnetic flux, the free energy, the current, and the Lorentz force for this event. (e)-(h) The magnetic structure of NLFFF extrapolations. (i)-(l) The evolution of the current. The red and blue patches show the positive and negative current. The green and black lines outline the sunspots with positive and negative polarities, respectively. (m)-(p) The evolution of the Lorentz force. The red and blue patches show the upward and downward Lorentz force. The blue arrows show the directions of the transverse Lorentz force.

which is tracked at the Carrington rotation rate (Sun 2013). The full set of time-dependent three-dimensional MHD equations is solved using the bottom boundary condition driven continuously by the changing photospheric vector magnetic fields from SDO observations (Jiang et al. 2016a). Here, the background plasma is initially in a hydrostatic isothermal state with $T=10^{6} \mathrm{~K}$ (sound speed $c_{S}=128 \mathrm{~km} \mathrm{~s}^{-1}$ ) in solar gravity. Its density is configured to make the plasma $\beta$ as small as $2 \times 10^{-3}$ (the maximum Alfvén $v_{\mathrm{A}}$ is $4 \mathrm{Mm} \mathrm{s}^{-1}$ ) to mimic the coronal low- $\beta$ and highly tenuous conditions. The plasma thermodynamics are simplified to be adiabatic since we focus on the evolution of the coronal magnetic field. The bottom boundary of the model is assumed to be the coronal base, and in addition, the magnetic field measured in the photosphere is used as a reasonable approximation to the field at the coronal base.

\section{Results}

A large active region in the southern hemisphere, named NOAA 12403, on 2015 August 24 (see Figure 1(a)) was located near the center of the solar disk with a $\beta \gamma \delta$ field configuration of the sunspot group. The $S D O$ observations lasted from the emergence of small satellite sunspots S1, S2, and S4 near the large sunspot with negative polarity to the occurrence of the flux rope eruption in this active region (see the positions of the satellite sunspots in Figures 2(a)-(d)). Moreover, BBSO highresolution observations detected the details of the eruption.

The evolution of the vector magnetic fields, NLFFF extrapolations, electric current, and Lorentz force during the formation and eruption of the flux rope is presented in Figure 2. The field of view of Figure 2 is marked by a yellow box in Figure 1(b). The emergence process of sunspots S1, S2, and S4 is clearly seen in the animation of Figure 8. Sunspot S2 first emerges to the right of sunspot S3 (see Figure 2(a)), and then it moves from south to north with a strong shearing motion between S2 and S3 (see Figures 2(b)-(c) and animation of Figure 8). The white and black patches indicate the positive and negative magnetic polarities in Figures 2(a)-(h). The blue arrows in Figures 2(a)-(d) indicate the transverse magnetic fields. The transverse magnetic fields around the four sunspots exhibit 


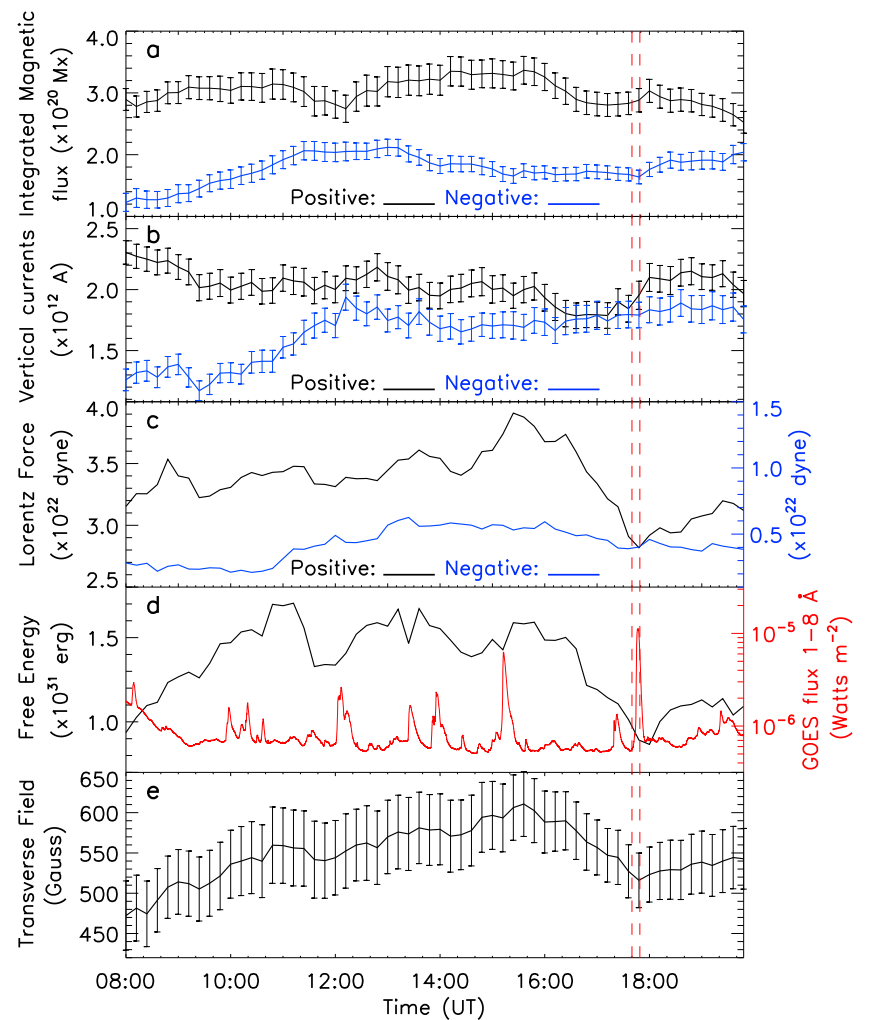

Figure 3. Evolution of the magnetic flux(a), the vertical current (b), the Lorentz force(c), the free energy (d), and the transverse magnetic fields (e). The red curve shows the GOES soft X-ray flux in panel (d). The vertical red dashed lines show the onset and ending time of the M-class flare.

clockwise vortex structure. The change in integrated magnetic flux in the white box of Figure 2(b) is shown in Figure 3(a). The NLFFF extrapolations in Figures $2(\mathrm{e})-(\mathrm{g})$ present the emergence of the structure of a flux rope, which includes the twisted magnetic field lines and a highly sheared arcade. Note that Space weather SDO/HMI Active Region Patches (SHARP) vector magnetogram data were used to extrapolate the three-dimensional magnetic field of this active region. After the eruption of the flux rope, the twisted structure disappeared and the connections of the magnetic field lines also changed (see Figure 2(h)). The configuration of magnetic fields became more potential.

Figures 2(i)-(l) show the evolution of the electric current during the emergence of the satellite sunspots and after the eruption of the flux rope. An increase in the current of sunspot S3 is associated with its emergence. The red and blue patches indicate the positive and negative electric current, respectively. The green and black contours outline the positive and negative magnetic polarities. Note that the levels of the contours are $\pm 500 \mathrm{G}, \pm 1300 \mathrm{G}$, and $\pm 2100 \mathrm{G}$. Since sunspots S2 and S3 exhibit a significant rotation during their evolution (see the animation of Figure 9), the direction of the Lorentz force also exhibits a vortex shape (see Figures $2(\mathrm{~m})-(\mathrm{p}))$. The rotation direction of the sunspots is consistent with the directions of the Lorentz force. During the emergence of sunspot S2, the upward and transverse Lorentz force between sunspots S2 and S3 increases rapidly from 07:36 UT to 11:12 UT.

Figure 3 presents the change in magnetic flux, vertical currents, Lorentz force, free energy, GOES X-ray flux, and transverse fields in the white box of Figure 2(b). The black and blue lines indicate the change in positive and negative magnetic flux in
Figure 3(a). It is significant that the negative magnetic flux increases during the emergence of sunspot S2, while the positive magnetic flux remains almost constant. The change in negative vertical currents is similar to the change in negative magnetic flux (see Figure 3(b)). The Lorentz force increases rapidly at 08:00 UT (the beginning of the emergence of sunspot S3) and at about 14:20 UT (three hours before onset of the flux rope eruption). The transverse fields and the Lorentz force reach their maximum values almost simultaneously (see Figures 3(c) and (e)). The positive vertical Lorentz force causes the flux rope to rise. Previous investigation indicates that the Lorentz force plays an important role in solar eruptions ( $\mathrm{Bi}$ et al. 2016). The transverse fields and the Lorentz force begin to decrease simultaneously at 15:20 UT. Because the transverse fields and Lorentz force are calculated in the photosphere, the decrease in these values suggests that the emergence of the field is now completed. The free magnetic energy begins to increase when the flux rope appears (see Figure 3(d)). After the emergence of the flux rope is completed, the decrease in free energy may be due to the eruption of some small chromospheric fibrils before the flux rope eruption.

Figure 4 shows the photospheric evolution of small satellite sunspots near the large sunspot observed by BBSO and SDO/ HMI. The TiO image and line-of-sight magnetogram observed by BBSO/NST and SDO/HMI are presented in Figures 4(a) and (b), respectively. Four small satellite sunspots are labeled S1, S2, S3, and S4 in Figures 4(a) and (b). All of the sunspots have positive polarity, except for $\mathrm{S} 2$, which has negative polarity. The four sunspots exhibit a clockwise rotation (see the animation of Figure 8). To obtain the rotation angle of sunspot S3, the circumference of a circle with a radius of $2^{\prime \prime}$ is mapped by a polar coordinate $\mathrm{r}-\theta$ frame centered on the middle of $\mathrm{S} 3$, as illustrated in Figure 4(c). The resolution used in the angular direction is $1^{\circ}$ to make the time slices (Zheng et al. 2016). The variation in rotation angle with time is calculated by tracing the penumbra of sunspot S3 (see Figure 4(f)). The green lines that trace two penumbral features show the rotation of penumbral filaments around the center of the circle. The rotation angles are $60^{\circ}$ after 42 minutes and $110^{\circ}$ after 200 minutes. The rotation of the sunspots implies that the twist is transferred from below the photosphere to the upper atmosphere (Sturrock \& Hood 2016). This is inferred from the increase of the twist in the flux rope that is associated with the rotation of the sunspot. Figures 4(c)-(e) demonstrate the evolution of these sunspots before and after the M-class flare. The M-class flare starts at 17:40 UT, peaks at 17:46 UT, and ends at 17:49 UT (see the red curve in Figure 3(d)). The penumbra between sunspot S2 and sunspot S3 gradually decreases before the onset of the M-class flare (see the blue arrows in Figures 4(c) and (d)). The disappearance of the penumbra is associated with the occurrence of the M-class flare (see Figure 4(e)). That is to say, the disappearance of the penumbra between sunspots $\mathrm{S} 2$ and $\mathrm{S} 3$ is due to the rising of the flux rope.

The entire eruptive process of the small flux rope was caught by the observation of BBSO/NST. Figure 5 shows the eruption process for the M-class flare observed by BBSO/ NST (see the animation of Figure 10). The three columns from left to right are the $\mathrm{H}$-alpha blue-wing ( $-0.6 \AA)$, $\mathrm{H}$-alpha center, and $\mathrm{H}$-alpha red-wing $(+0.6 \AA)$ images. The contours superimposed on the first-row images are the levels of the line-of-sight magnetic fields. The green and blue contours indicate positive and negative magnetic polarity. The levels of the contours are $\pm 300 \mathrm{G}$ and $\pm 800 \mathrm{G}$. The red 

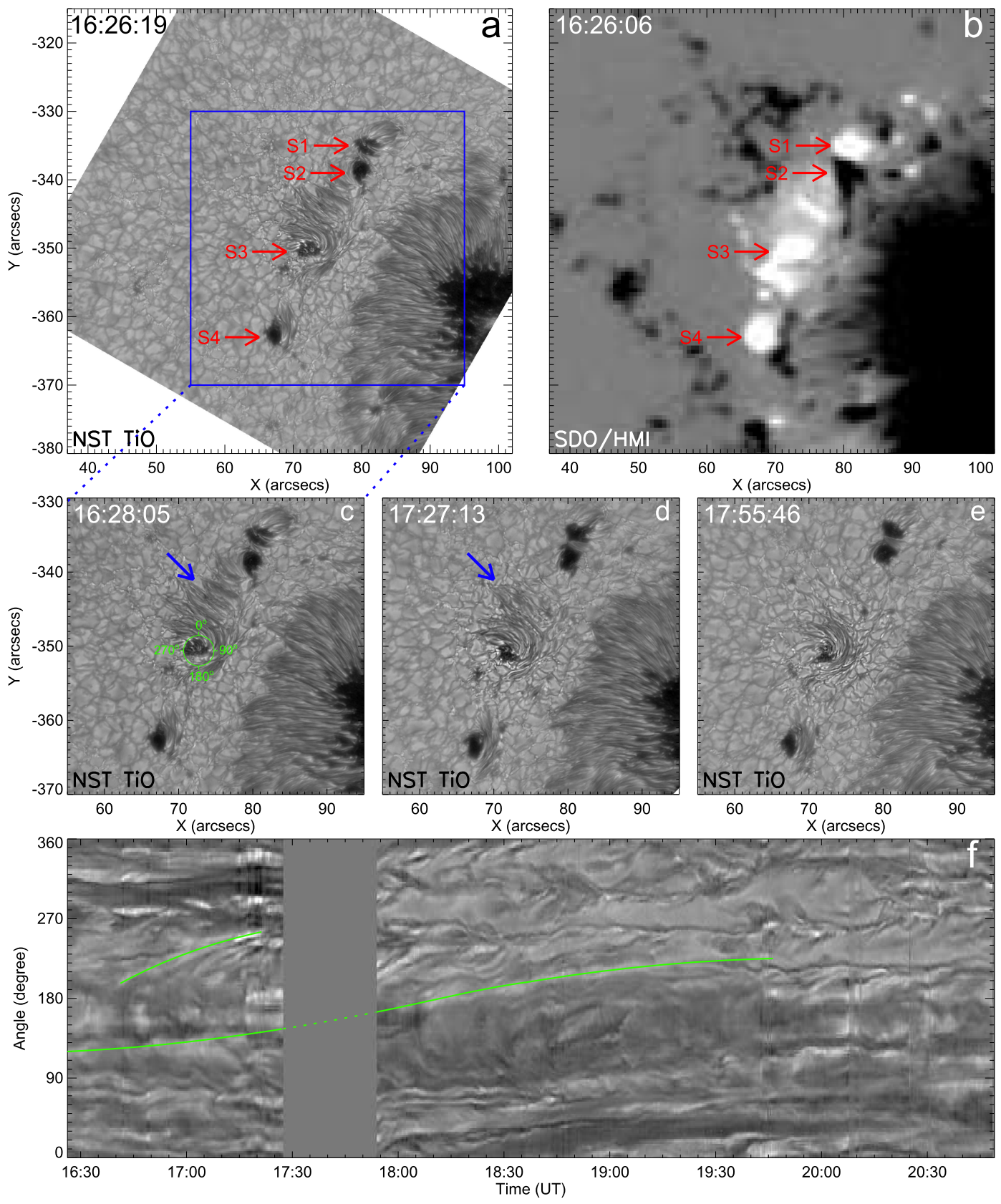

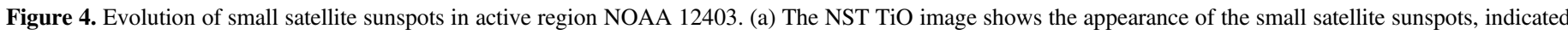

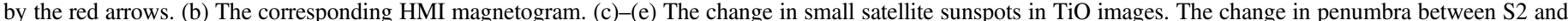

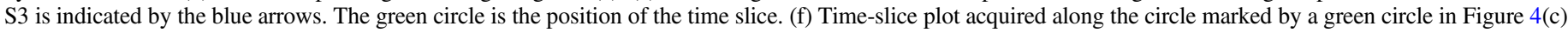
and the green curved lines indicate the rotation of the penumbra filament around the center of sunspot S3.

arrows mark the positions of the sunspots. The yellow arrows in Figures 5(a)-(c) indicate the flux rope before its eruption. It is clear that the two footpoints of the flux rope are mainly rooted in sunspots $\mathrm{S} 2$ and $\mathrm{S} 3$. Some threads of the flux rope also seen to be rooted in the outer penumbra of the large sunspot, which is implied by the NLFFF extrapolations (see Figures 2(e), (f), and (g)). Before the flux rope eruption, a series of small chromospheric fibrils at the upper end of the flux rope first begin to erupt from 16:37:19 UT to 17:00:37 UT (see the green arrows in Figures 5(d)-(i)). At 17:09:05 UT, the upper part of the flux rope becomes darker and starts to erupt. A strong blueshift can be seen in the $\mathrm{H}$-alpha blue-wing images. The closed magnetic field lines are opened and followed by a strong redshift in the $\mathrm{H}$-alpha red-wing images. The flux rope starts to erupt at 17:40:12 UT, associated with a strong redshift at the upper end of the flux rope. At the beginning of the flux rope eruption, the whole body of the flux rope rotated counterclockwise. After the eruption of the flux rope, the closed magnetic field lines are opened again (see the $\mathrm{H}$-alpha blue-wing and the $\mathrm{H}$-alpha center observation at about 17:50:40 UT). These observations clearly show that before the onset of the large eruption, small eruptions of chromospheric fibrils occur as a precursor. Using observational data from the Swedish $1 \mathrm{~m}$ Solar Telescope (SST) and Hinode, Guglielmino et al. (2010) found that reconnection between the small-scale emerging flux region and the overlying coronal field produces the brightenings in the chromosphere, transition region, and corona, as well as 

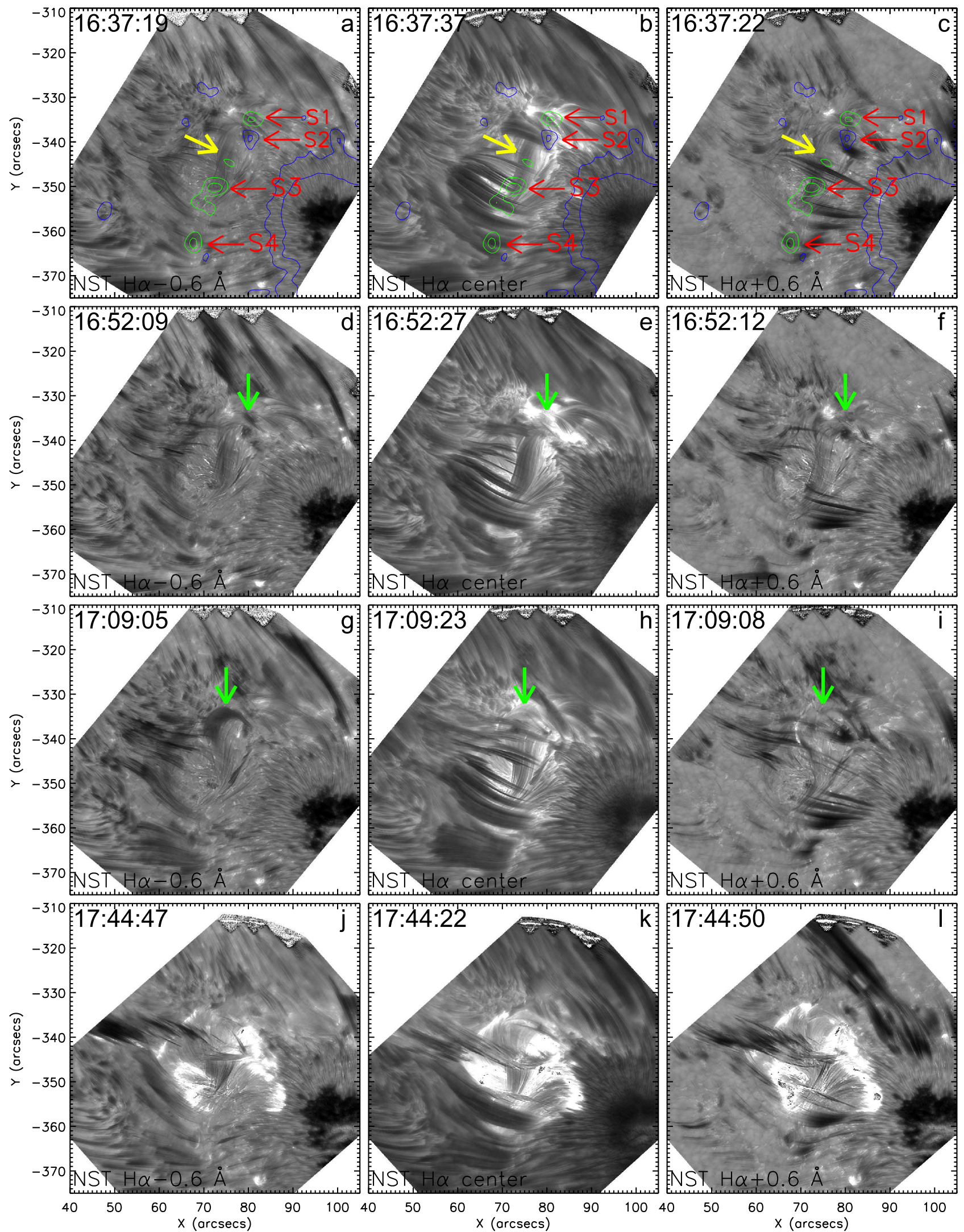

Figure 5. Eruption process of the M-class flare. (a), (d), (g), and (j): the NST H-alpha blue-wing images acquired at -0.6 A. (b), (e), (h), and (k): the NST H-alpha center images. (c), (f), (i), and (l): the NST H-alpha red-wing images acquired at $+0.6 \AA$. The first-row images are overlaid by green (blue) contours representing positive (negative) polarity. The yellow arrows indicate the flux rope, and the red arrows indicate the small satellite sunspots. The green arrows indicate the small threads at the upper part of the flux rope, which erupt before the flux rope eruption. 

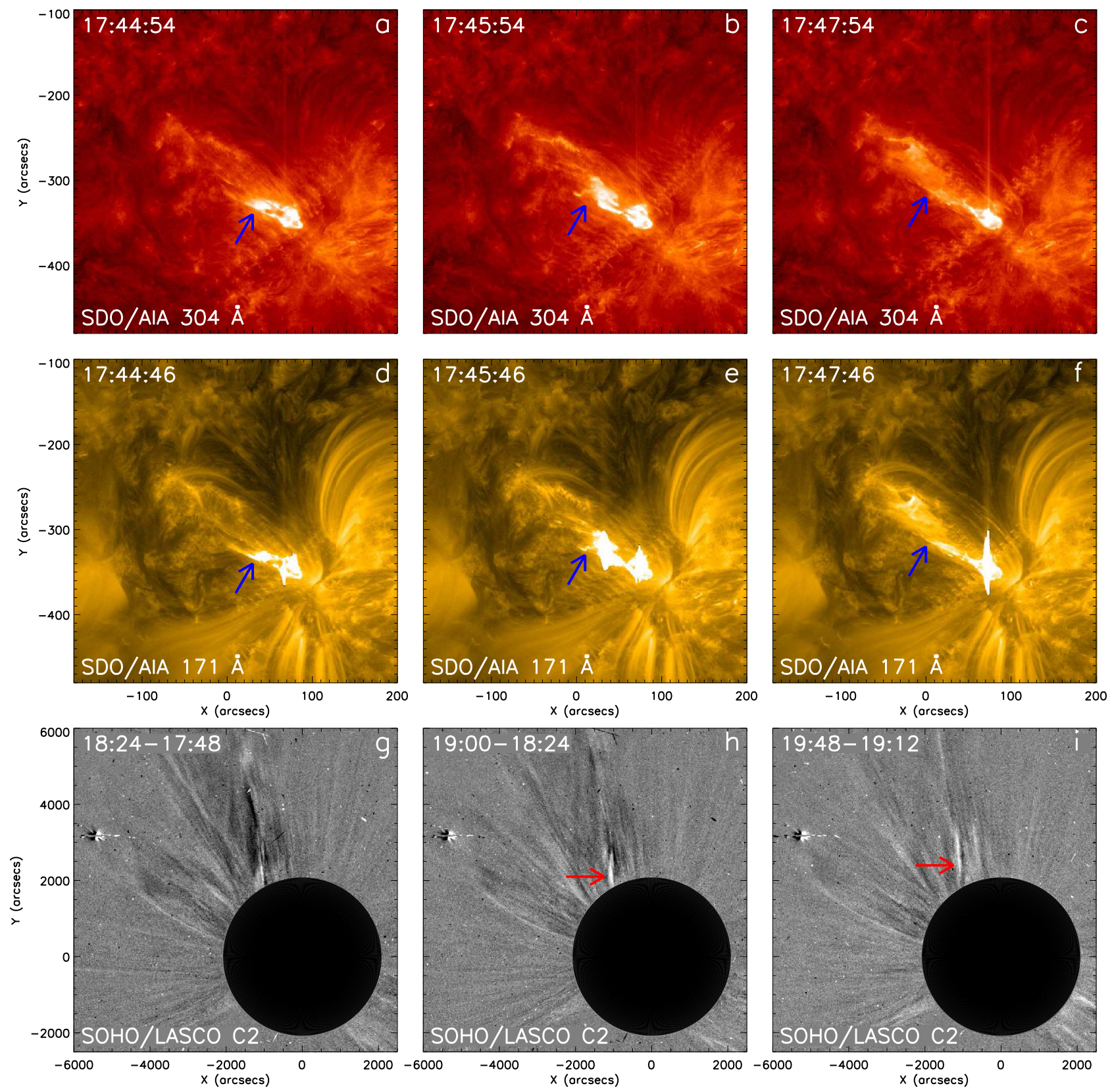

Figure 6. M-class flare and CME observed in SDO $304 \AA$ and $171 \AA$ images, and SOHO/LASCO C2. The blue arrows indicate the plasma ejection from the active region, while the red arrows indicate the small coronal mass ejection after the flux rope eruption.

chromospheric surges. Our results are very similar to these observations. The change in topology in our study is also similar to what is found in flux emergence simulations when the emerging field pushes into an overlying field and reconnects with it (Galsgaard et al. 2005; Leake et al. 2014; MacTaggart \& Haynes 2014). The precursor of a flux rope eruption is also similar to the simulation results of MacTaggart et al. (2015), which show how the surges occur when a small-scale emerging flux region reconnects with ambient fields.

During the flux rope eruption, the plasma is ejected into the upper atmosphere (see Figures 6(a)-(f)). The blue arrows in Figures 6(a)-(f) indicate the flux rope during its eruption in $304 \AA$ and $171 \AA$ images. At 18:24 UT, LASCO C2 observed a coronal mass ejection (see Figures 6(g)-(i)).

A data-driven MHD model for solar active-region evolution (Jiang et al. 2016a) is employed to provide further understanding of the process of emergence and eruption of the field, which is shown in Figure 7. Here the MHD simulation starts at 08:00 UT on August 24, when the small sunspot S2 just emerges near the small sunspot $\mathrm{S} 3$. The initial conditions consist of an NLFFF extrapolated from the vector magnetogram at this start time and a highly tenuous plasma in a hydrostatic state. Subsequently, the model is continuously supplied with a changing bottom boundary condition from the data stream of photospheric vector magnetograms observed by $S D O / \mathrm{HMI}$. To self-consistently input the vector magnetograms at the boundary, the method of projected characteristics based on the wave-decomposition principle of the full MHD system (Wu et al. 2006) was used. The result of the simulation can be seen in Figures 7(a)-(c) and in the animation of Figure 11. The evolution of the electric current in a cross section along (see Figures 7(d)-(f)) and perpendicular (see Figures 7(g)-(i)) to the flux rope is calculated 

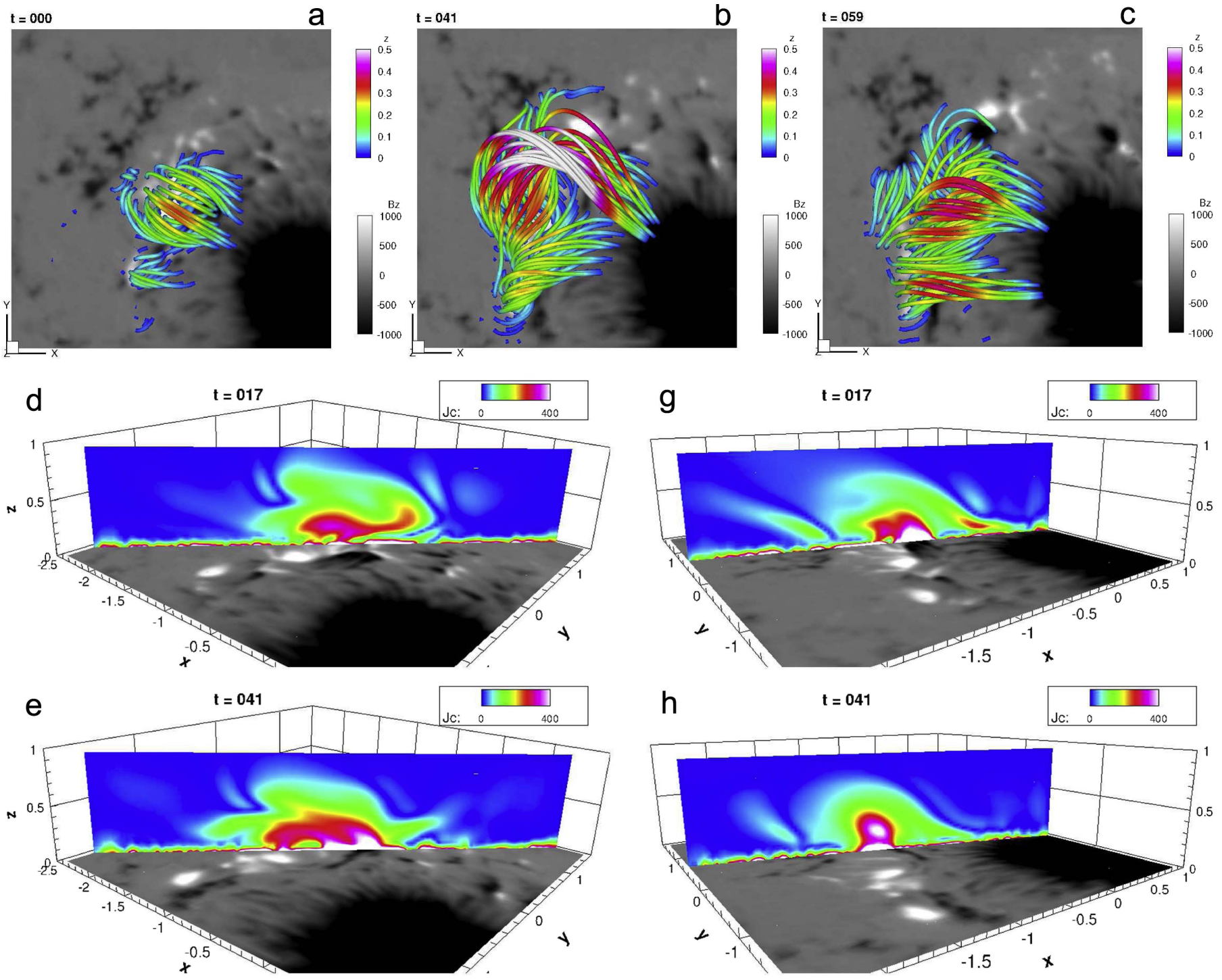

$\mathrm{h}$

$\mathbf{t}=041$
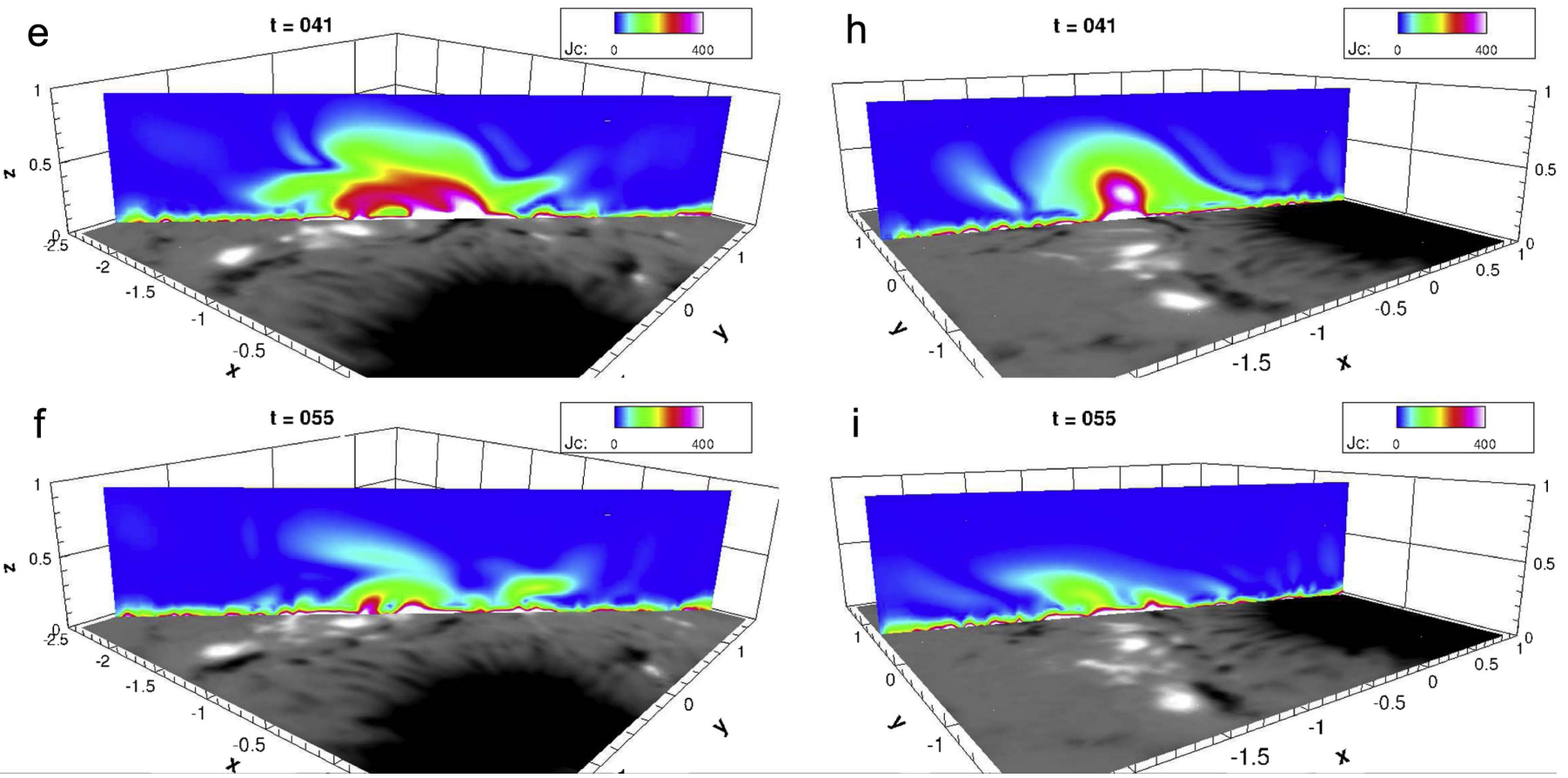

Figure 7. Data-driven MHD simulation of flux rope emergence and eruption. (a)-(c): emergence of the flux rope. (d)-(f): current along the flux rope. (g)-(i): current perpendicular to the flux rope.

from the simulation. It is clear that the magnetic field emerges in the course of the simulation from $t=0$ to 41 , as driven by the evolution of the photospheric field. During the emergence of the field, the current increases rapidly along the flux rope, and the rising of the flux rope can also be seen from the cross section of the current perpendicular to the axis of the flux rope. At around $t=55$, both the twisted magnetic flux and the corresponding current density decreased quickly, indicating the eruption of the flux rope, which released some of the non-potential energy of the pre-eruption field, after which the magnetic structure is closer to potential. These observation and simulation confirm the existence of flux rope in the solar eruptions (Xue et al. 2016).

\section{Conclusions and Discussions}

Observational evidence of the emergence of a small-scale flux rope as the trigger of the M-class flare and a CME were present using high spatial and temporal data from NST and 


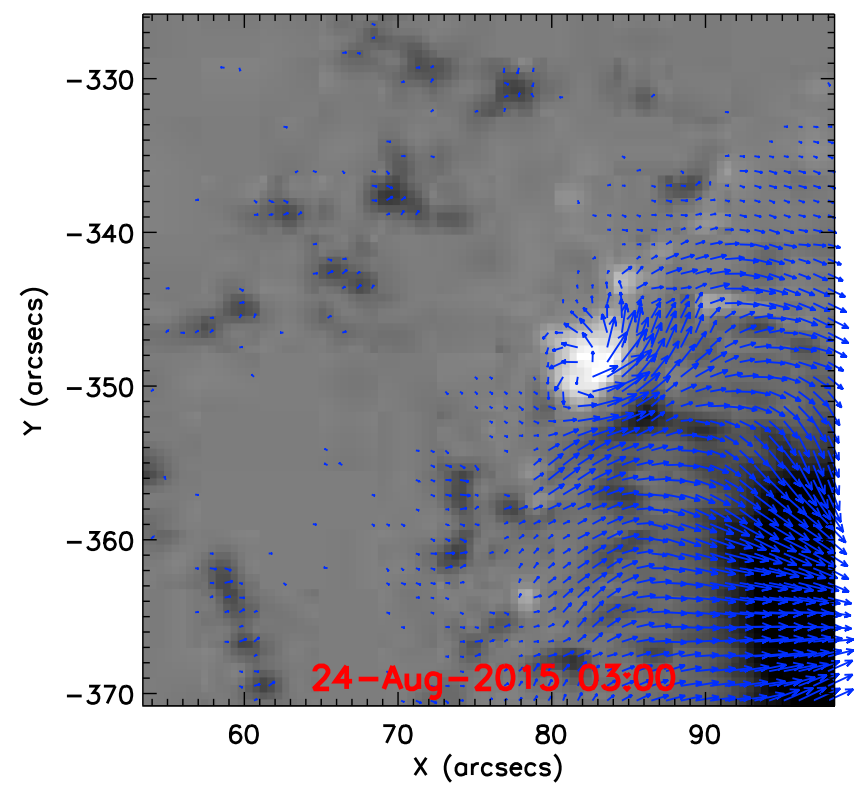

Figure 8. Online animation of vector magnetograms observed by $S D O / \mathrm{HMI}$ as shown in Figure 2. The $4 \mathrm{~s}$ duration animation covers $24 \mathrm{hr}$ on 2015 August 24. (An animation of this figure is available.)

SDO. The small sunspot with negative polarity emerges near the small sunspot with positive polarity. During the emergence of the small-scale flux rope, the negative flux, the electric current, the Lorentz force, and the transverse magnetic fields increase gradually. The magnetic free energy calculated from the extrapolated three-dimensional magnetic fields is found to be enough to power the M-class flare. The NLFFF extrapolation and the data-driven MHD simulation also reveal the emergence of the flux rope. Moreover, the sunspots in which the flux rope was rooted exhibit clockwise rotation. These observations present a clear picture of solar eruptions: emergence of the small-scale flux rope leads to the flare and the CME.

There are several pieces of evidence that can confirm the emergence of the flux rope. First, the small sunspot with negative polarity emerged near the small sunspot with positive polarity. The negative magnetic fluxes and the electric current obviously increase at the emergence of the flux rope. Most of magnetic field lines of the flux rope extrapolated from the NLFFF were rooted in the two small sunspots. Second, the transverse magnetic fields increase at first and decrease about two hours before its eruption. Third, the change in upward Lorentz force also increases at the beginning of the flux rope and then decrease before the eruption of the flux rope, which is similar to that of the transverse of the magnetic fields. Fourth, the sunspot rotation was observed during the emergence of the flux rope. Fifth, the twisted magnetic structures can be obtained from the NLFFF extrapolation. Sixth, the data-driven MHD modeling of the dynamic evolution of the coronal three-dimensional magnetic field recreates the emergence of the flux rope. Seventh, the free magnetic energy increases rapidly at the stage of the flux rope emergence. We observed sunspot rotation, shearing between the two opposite polarities, and the separation of the two small sunspots during emergence of the field. This scenario is very consistent with simulations of the emergence of a twisted flux tube from the subphotosphere to the corona (Magara 2006; Fan 2009; Hood et al. 2009, 2012; Archontis et al. 2014).
Several surge-like eruptions were observed as the precursor of the eruption of the small-scale twisted flux rope. This agrees well with the prediction from emerging flux simulations of Galsgaard et al. (2005) and MacTaggart et al. (2015). Furthermore, as the flux rope emerges, the Lorentz force produces shearing along the polarity inversion line between the two small sunspots, and the flux rope is carried upward by the positive Lorentz force. These observations match the simulation of MacTaggart \& Hood (2009) very well.

Helical flux ropes are fundamental magnetic structures in some eruptive-flare models (Priest et al. 1989; van Ballegooijen \& Martens 1989; Rust \& Kumar 1994; Amari et al. 2003; Gibson et al. 2006; Fan 2009). However, it is very difficult to determine whether the flux ropes form before or during eruptions. Much observational evidence supports the hypothesis that flux ropes already exist before the onset of solar eruptions (Liu et al. 2003; Yan et al. 2012; Chintzoglou et al. 2015; Su et al. 2015; Cheng \& Ding 2016; Vemareddy et al. 2016; James et al. 2017). Others suggest that flux ropes form during solar eruptions (Song et al. 2014; Cheng et al. 2010). Recent observations show that unwinding motion is often found during active-region filament eruptions (Yan et al. 2014a, 2014b; Zhang et al. 2015b), which implies that the active-region filaments may have twisted magnetic structures. Srivastava et al. (2010) presented direct observational evidence for the existence of a twisted magnetic structure in the corona. Owing to the spatial and temporal resolution of the data, where the twist of flux ropes comes from is not easy to observe, sunspot rotation is one possible way for injecting magnetic twists into the corona, and the twisted magnetic structures or solar filaments are indeed observed after sunspot rotation (Yan et al. 2012, 2015; James et al. 2017). In some cases, flux ropes are formed by magnetic reconnection of sheared arcade loops (Chen et al. 2014; Yang et al. 2016; Wang et al. 2017; Xue et al. 2017). Another way is the emergence of twisted flux ropes from below the photosphere in many simulations (Magara 2006; Fan 2009; Hood et al. 2009; MacTaggart \& Hood 2009; Hood et al. 2012).

The formation process of small-scale flux ropes cannot normally be observed because we lack high-resolution photospheric magnetograms and chromospheric observations. Fortunately, the observations of NST and SDO caught a clear process of the emergence of a small flux rope. In this study, the photospheric and chromospheric observations of NST, supplemented by $S D O$ observations, can present the whole process of the formation of a flux rope associated with the emergence of a small sunspot (pore). Moreover, the two small sunspots at the footpoints of the flux rope exhibited a clockwise rotation. Sunspot S2 rotates around its center and moves away from sunspot S3 during emergence of the flux rope. This scenario is consistent with the emergence of a twisted flux rope. The increase in twist in the flux rope with time follows the rotation of small sunspots. It implies that sunspot rotation may play an important role in the increase of the twist of the flux rope, which propagates from below the photosphere to the upper atmosphere. In the past, many simulations have included high twist to generate flux ropes and CMEs in the atmosphere. Our results tie in well with such simulations (Galsgaard et al. 2005; Magara 2006; Fan 2009; Hood et al. 2009; MacTaggart \& Hood 2009; Hood et al. 2012; MacTaggart et al. 2015). Our observations give very strong evidence for small-scale highly twisted ropes emerging to form CMEs, as the simulations predict. 

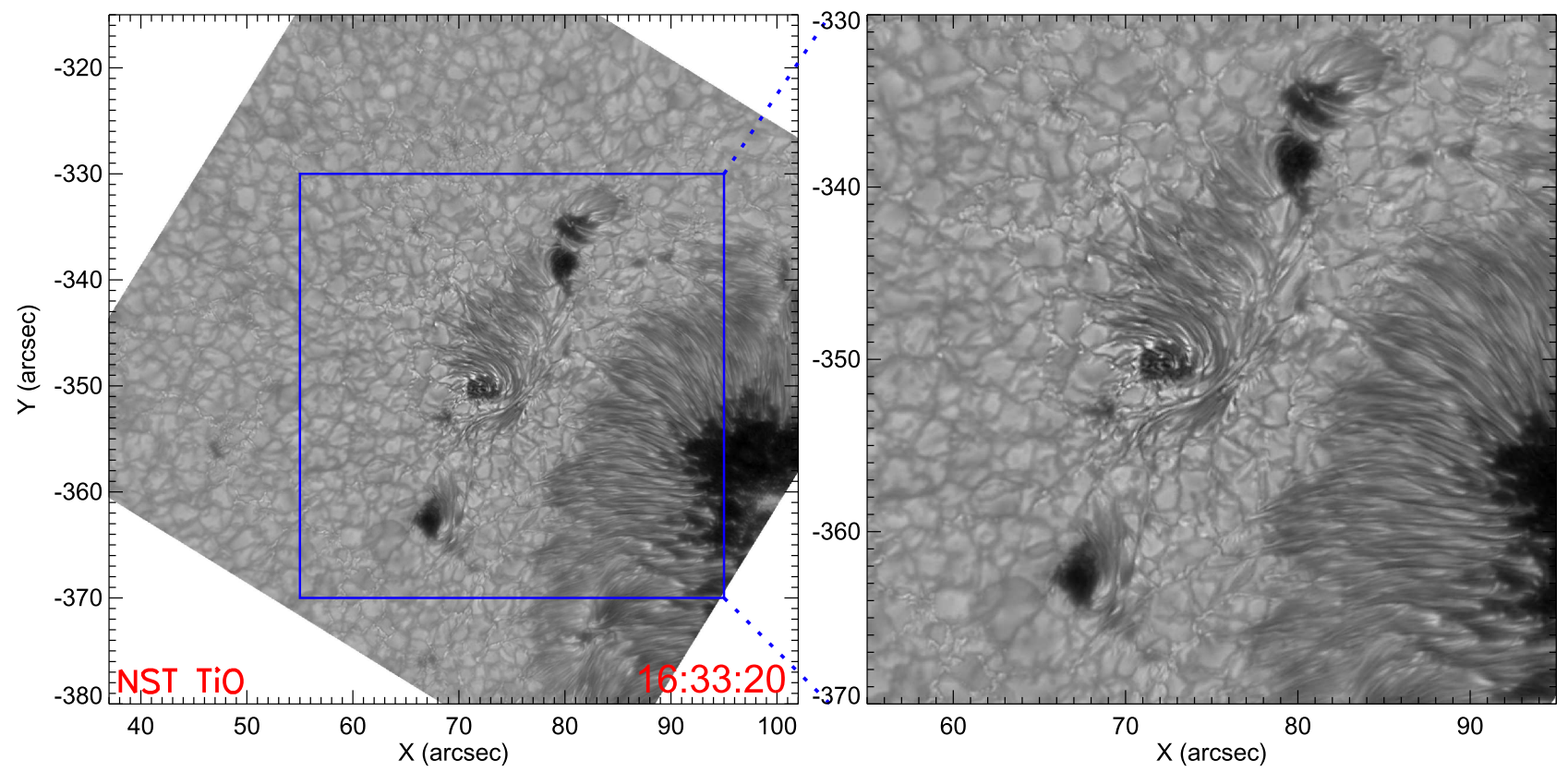

Figure 9. Online animation of the evolution of small satellite sunspots in active region NOAA 12403 with NST TiO as shown in Figure 4 . The $16 \mathrm{~s}$ duration animation covers $\sim 4.5 \mathrm{hr}$ from 16:26 to 20:50 UT.

(An animation of this figure is available.)
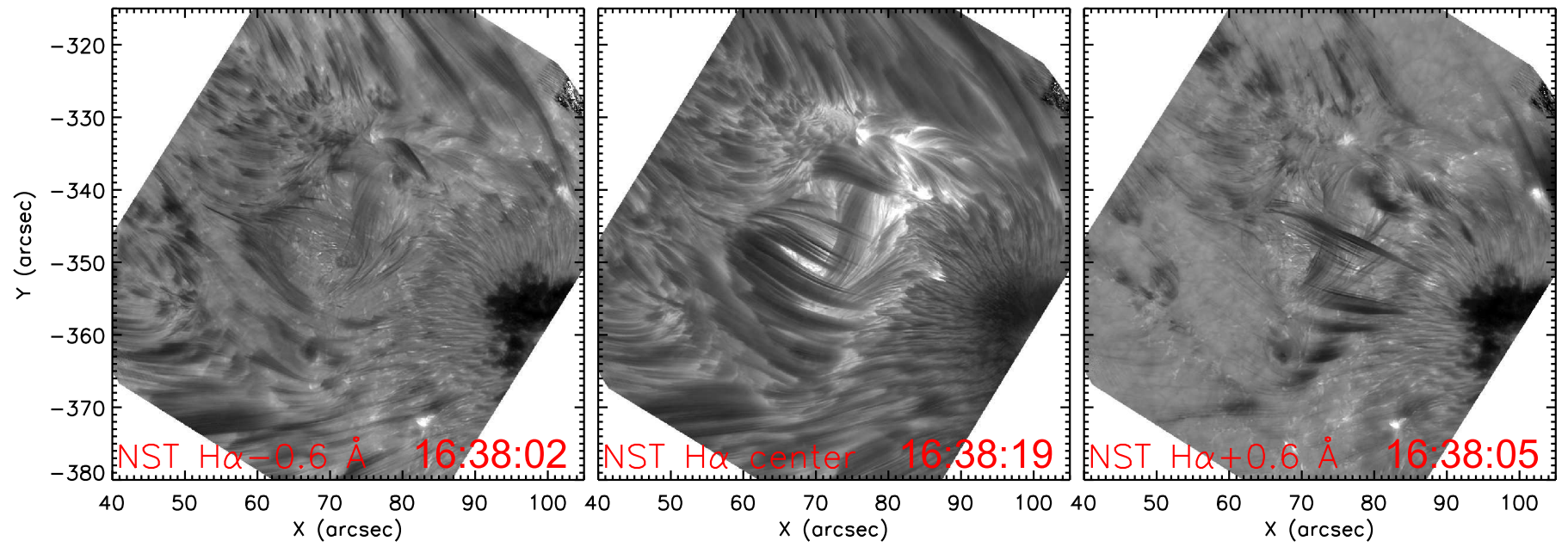

Figure 10. Online animation of the NST H-alpha blue-wing, center, and red-wing images shown in Figure 5. The $6 \mathrm{~s}$ animation covers $\sim 2 \mathrm{hr}$ from 16:25 to $18: 35 \mathrm{UT}$. (An animation of this figure is available.)

The eruptive mechanism for solar eruptions is also an open issue. Several models have been developed to address this question. Ideal MHD instability is one of these models (Hood \& Priest 1979). Török \& Kliem 2003 simulated the evolutionary process of a twisted flux rope and found that when the critical value of twist is higher than $2.75 \pi$, the flux rope cannot keep stable and will erupt. Some observations can also confirm their results (Srivastava et al. 2010; Kumar et al. 2012; Yan et al. 2014a, 2014b). In this study, the twist of the flux rope extrapolated from NLFFF is about three turns. When the smallscale magnetic region emerged from below the penumbra of the large sunspot, the overlying magnetic field was perpendicular to the axis of the flux rope. The flux rope exhibited a counterclockwise rotation at the onset of its eruption, however, and the axis of the flux rope became antiparallel to the overlying magnetic field. External reconnection between the emerging field and the overlying magnetic field may weaken the tension of the coronal field. This scenario is very similar to the simulations of MacTaggart \& Hood (2009) and Leake et al. (2014). Therefore, reconnection between the emerging field and the preexisting extended field of the large sunspot also plays an important role in flux rope eruption.

We would like to thank the NST, SDO/AIA, and SDO/HMI teams for the high-cadence data support. This work is sponsored by the National Science Foundation of China (NSFC) under the grant numbers 11373066, 11603071, 11503080, 11633008, 11533008, by the Key Laboratory of Solar Activity of CAS under numbers KLSA201603, KLSA201508, by the Yunnan Science Foundation of China under number 2013FB086, CAS "Light of West China" 


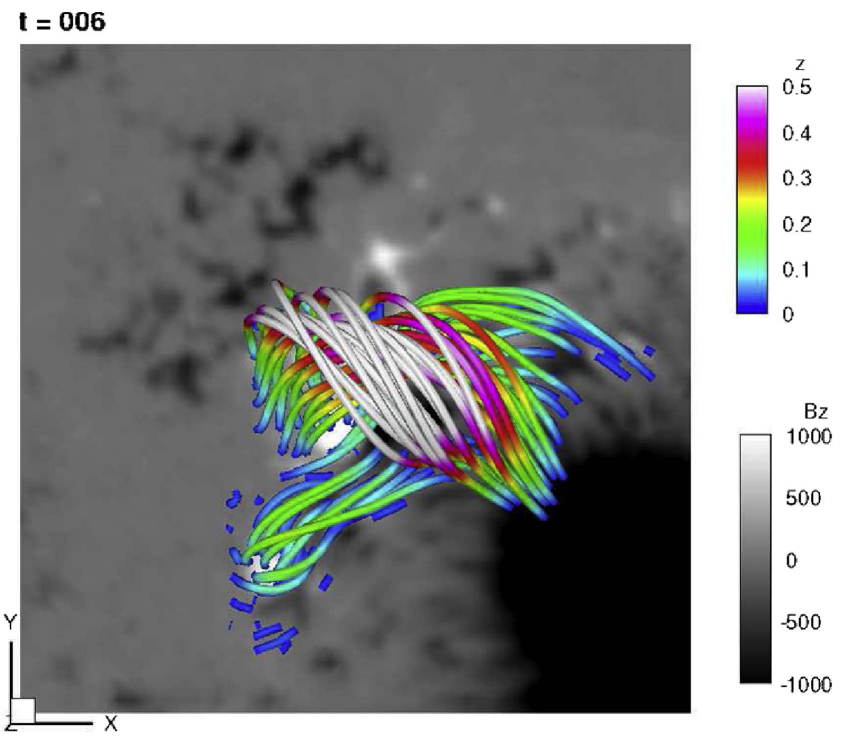

Figure 11. Online animation of the data-driven MHD simulation of flux rope emergence and eruption shown in Figure 7. The $6 \mathrm{~s}$ animation covers 60 timesteps of the simulation.

(An animation of this figure is available.)

Program, by the Youth Innovation Promotion Association CAS (No.2011056), and the national basic research program of China (973 program, 2011CB811400). The BBSO operation is supported by NJIT, US NSF AGS-1250818, and NASA NNX13AG14G grants, and the NST operation is partly supported by the Korea Astronomy and Space Science Institute and Seoul National University and by the strategic priority research program of CAS with Grant No. XDB09000000.

\section{ORCID}

C. W. Jiang (10) https://orcid.org/0000-0002-7018-6862

E. R. Priest (iD https://orcid.org/0000-0003-3621-6690

\section{References}

Amari, T., Luciani, J. F., Aly, J. J., Mikic, Z., \& Linker, J. 2003, ApJ, 585,1073

Antiochos, S. K., DeVore, C. R., \& Klimchuk, J. A. 1999, ApJ, 510, 485

Archontis, V., \& Hood, A. W. 2013, ApJ, 769, 21

Archontis, V., Hood, A. W., \& Tsinganos, K. 2014, ApJ, 786, 21

Bi, Y., Jiang, Y., Yang, J., et al. 2016, NatCo, 7, 13798

Bobra, M. G., Sun, X., Hoeksema, J. T., et al. 2014, SoPh, 289, 3549

Borrero, J. M., Tomczyk, S., Kubo, M., et al. 2011, SoPh, 273, 267

Cao, W., Gorceix, N., Coulter, R., et al. 2010, AN, 331, 636

Carmichael, H. 1964, NASSP, 50, 451

Centeno, R., Schou, J., Hayashi, K., et al. 2014, SoPh, 289, 3531

Chen, H., Zhang, J., Cheng, X., et al. 2014, ApJ, 797, 15

Chen, P. F., \& Shibata, K. 2000, ApJ, 545, 524

Chen, Y., Du, G., Zhao, D., et al. 2016, ApJ, 820, 37

Cheng, X., \& Ding, M. D. 2016, ApJS, 225, 16

Cheng, X., Ding, M. D., Guo, Y., et al. 2010, ApJ, 716, 68

Chintzoglou, G., Patsourakos, S., \& Vourlidas, A. 2015, ApJ, 809, 34

Deng, L. H., Qu, Z. Q., Liu, T., \& Wang, K. R. 2013, AN, 334, 217

Fan, Y. 2009, ApJ, 697, 1529

Filippov, B., Martsenyuk, O., Srivastava, A. K., \& Uddin, W. 2015, JApA, 36, 157 Fisher, G. H., Bercik, D. J., Welsch, B. T., \& Hudson, H. S. 2012, SoPh, 277, 59

Forbes, T. G. 2000, JGR, 105, 23153

Galsgaard, K., Moreno-Insertis, F., Archontis, V., \& Hood, A. 2005, ApJ, 618,153

Gibson, S. E., Fan, Y., Török, T., \& Kliem, B. 2006, SSRv, 124, 131

Goode, P. R., \& Cao, W. D. 2010, Proc. SPIE, 8444, 03
Guglielmino, S. L., Bellot Rubio, L. R., Zuccarello, F., et al. 2010, ApJ, 724, 1083

Hirayama, T. 1974, SoPh, 34, 323

Hood, A. W., Archontis, V., Galsgaard, K., \& Moreno-Insertis, F. 2009, A\&A, 503,999

Hood, A. W., Archontis, V., \& MacTaggart, D. 2012, SoPh, 278, 3

Hood, A. W., \& Priest, E. R. 1979, SoPh, 64, 303

Hou, Y. J., Li, T., \& Zhang, J. 2016, A\&A, 592, 138

James, A. W., Green, L. M., Palmerio, E., et al. 2017, SoPh, 292, 71

Jiang, C. W., Wu, S. T., Feng, X. S., \& Hu, Q. 2014, ApJ, 786, 16

Jiang, C. W., Wu, S. T., Feng, X. S., \& Hu, Q. 2016a, NatCo, 7, 11522

Jiang, C. W., Wu, S. T., Feng, X. S., \& Hu, Q. 2016b, RAA, 16, 18

Joshi, N. C., Magara, T., \& Inoue, S. 2014, ApJ, 795, 4

Kliem, B., Lin, J., Forbes, T. G., Priest, E. R., \& Török, T. 2014, ApJ, 789,46

Kliem, B., \& Török, T. 2006, PhRvL, 96, 255002

Kopp, R. A., \& Pneuman, G. W. 1976, SoPh, 50, 85

Kumar, P., Cho, K. S., Bong, S. C., Park, S. H., \& Kim, Y. H. 2012, ApJ, 746, 67

Kumar, P., Yurchyshyn, V., Cho, K., \& Wang, H. 2017, A\&A, 603, A36

Leake, J. E., Linton, M. G., \& Antiochos, S. K. 2014, ApJ, 787, 46

Leka, K. D., Barnes, G., Crouch, A. D., et al. 2009, SoPh, 260, 83

Lemen, J. R., Title, A. M., Akin, D. J., et al. 2012, SoPh, 275, 17

Li, L, Zhang, J., Su, J. T., \& Liu, Y. 2016a, ApJ, 829, 33

Li, T., Yang, K., Hou, Y. J., \& Zhang, J. 2016b, ApJ, 830, 152

Lim, E. K., Yurchyshyn, V., Park, S. H., et al. 2016, ApJ, 817, 39

Lin, J., \& Forbes, T. G. 2000, JGR, 105, 2375

Lin, J., Murphy, N., Shen, C., et al. 2015, SSRv, 194, 237

Liu, Y., Jiang, Y., Ji, H., Zhang, H., \& Wang, H. 2003, ApJ, 593, 137

Liu, Z., Xu, J., Gu, B. Z., et al. 2014, RAA, 14, 705

MacTaggart, D., Guglielmino, S. L., Haynes, A. L., Simitev, R., \& Zuccarello, F. 2015, A\&A, 576, 4

MacTaggart, D., \& Haynes, A. L. 2014, MNRAS, 438, 1500

MacTaggart, D., \& Hood, A. W. 2009, A\&A, 508, 445

Magara, T. 2006, ApJ, 653, 1499

Metcalf, T. R. 1994, SoPh, 155, 235

Metcalf, T. R., Leka, K. D., Barnes, G., et al. 2006, SoPh, 237, 267

Moore, R. L., Falconer, D. A., \& Sterling, A. C. 2012, ApJ, 750, 24

Moore, R. L., Sterling, A. C., Hudson, H. S., \& Lemen, J. R. 2001, ApJ, 552,833

Moreno-Insertis, F., \& Galsgaard, K. 2013, ApJ, 771, 20

Nóbrega-Siverio, D., Moreno-Insertis, F., \& Martínez-Sykora, J. 2016, ApJ, 822,18

Okamoto, T. J., Tsuneta, S., Lites, B. W., et al. 2009, ApJ, 697, 913

Priest, E. R., \& Forbes, T. G. 2002, A\&AR, 10, 313

Priest, E. R., Hood, A. W., \& Anzer, U. 1989, ApJ, 344, 1010

Priest, E. R., \& Longcope, D. W. 2017, SoPh, 292, 25

Régnier, S., \& Canfield, R. C. 2006, A\&A, 451, 319

Rust, D. M., \& Kumar, A. 1994, SoPh, 155, 69

Rust, D. M., \& Kumar, A. 1996, ApJ, 646, L199

Schmieder, B., Aulanier, G., \& Vrsnak, B. 2015, SoPh, 290, 3457

Schou, J., Scherrer, P. H., Bush, R. I., et al. 2012, SoPh, 275, 229

Schrijver, C. J., Elmore, C., Kliem, B., Török, T., \& Title, A. M. 2008, ApJ, 674,586

Shen, J. H., Wang, Y., Zhou, T. H., \& Ji, H. S. 2017, ApJ, 835, 43

Shen, Y., Liu, Y., \& Su, J. 2012, ApJ, 750, 12

Shibata, K., Masuda, S., Shimojo, M., et al. 1995, ApJ, 451, 83

Song, H. Q., Chen, Y., Zhang, J., et al. 2015, ApJL, 808, L15

Song, H. Q., Zhang, J., Chen, Y., \& Cheng, X. 2014, ApJ, 792, 40

Srivastava, A. K., Zaqarashvili, T. V., Kumar, P., \& Khodachenko, M. L. 2010, ApJ, 715, 292

Sterling, A. C., Chifor, C., Mason, H. E., Moore, R. L., \& Young, P. R. 2010, A\&A, 521, 49

Sturrock, P. A. 1966, Natur, 211, 695

Sturrock, Z., \& Hood, A. W. 2016, A\&A, 593, A63

Sturrock, Z., Hood, A. W., Archontis, V., \& McNeill, C. M. 2015, A\&A, 582,76

Su, Y. N., van Ballegooijen, A., McCauley, P., et al. 2015, ApJ, 807, 144

Sun, X. 2013, arXiv:1309.2392

Tian, H., Yao, S., Zong, Q., He, J., \& Qi, Y. 2010, ApJ, 720, 454

Török, T., \& Kliem, B. 2003, A\&A, 406, 1043

Török, T., Kliem, B., \& Titov, V. S. 2004, A\&A, 413, L27

van Ballegooijen, A. A., \& Martens, P. C. H. 1989, ApJ, 343, 971

Vargas Dominguez, S., MacTaggart, D., Green, L., van Driel-Gesztelyi, L., \& Hood, A. W. 2012, SoPh, 278, 33

Vemareddy, P., Cheng, X., \& Ravindra, B. 2016, ApJ, 829, 24

Wang, H. M., Cao, W. D., Liu, C., Yan, X., et al. 2015, NatCo, 7, 7008 
Wang, H. M., Ewell, M. W., Zirin, H., \& Ai, G. X. 1994, ApJ, 424, 436

Wang, J. C., Yan, X. L., Qu, Z. Q., Xue, Z. K., \& Yang, L. H. 2017, ApJ, 839, 128

Wang, J. X., \& Shi, Z. X. 1993, SoPh, 143, 119

Wang, Y. M., Ye, P. Z., Wang, S., Zhou, G. P., \& Wang, J. X. 2002, JGR, 107,1340

Wheatland, M. S., Sturrock, P. A., \& Roumeliotis, G. 2000, ApJ, 540, 1150

Wiegelmann, T. 2004, SoPh, 219, 87

Wiegelmann, T., Inhester, B., \& Sakurai, T. 2006, SoPh, 233, 215

Wu, S. T., Wang, A. H., Liu, Y., \& Hoeksema, J. T. 2006, ApJ, 652, 800

Xiang, N. B., \& Qu, Z. N. 2016, AJ, 151, 76

Xue, Z. K., Yan, X. L., Cheng, X., et al. 2016, NatCo, 7, 11837

Xue, Z. K., Yan, X. L., Yang, L. H., Wang, J. C., \& Zhao, L. 2017, ApJL, 840, L23

Yan, X. L., Priest, E. R., Guo, Q. L., et al. 2016, ApJ, 832, 23

Yan, X. L., \& Qu, Z. Q. 2007, A\&A, 468, 1083

Yan, X. L., Qu, Z. Q., Kong, D. F., \& Xu, C. L. 2012, ApJ, 754, 16
Yan, X. L., Qu, Z. Q., Xu, C. L., Xue, Z. K., \& Kong, D. F. 2009, RAA, 9, 596 Yan, X. L., Xue, Z. K., Liu, J. H., et al. 2014a, ApJ, 782, 67

Yan, X. L., Xue, Z. K., Liu, J. H., Kong, D. F., \& Xu, C. L. 2014b, ApJ, 797, 52

Yan, X. L., Xue, Z. K., Pan, G. M., et al. 2015, ApJS, 219, 17

Yang, B., Jiang, Y., Yang, J., Bi, Y., \& Li, H. 2016, ApJ, 830, 16

Yang, B., Jiang, Y., Yang, J., Hong, J., \& Xu, Z. 2015, ApJ, 803, 86

Yang, S. H., Zhang, J., Liu, Z., \& Xiang, Y. Y. 2014, ApJ, 784, L36

Zhang, J., Cheng, X., \& Ding, M. D. 2012, NatCo, 3, 747

Zhang, J., Li, L., \& Song, Q. 2007, ApJ, 662, 35

Zhang, J., Yang, S. H., \& Li, L. 2015a, A\&A, 580, 2

Zhang, Q. M., Ning, Z. J., Guo, Y., et al. 2015b, ApJ, 805, 4

Zheng, J., Yang, Z., Guo, J., et al. 2017, RAA, 17, 81

Zheng, J. C., Yang, Z. L., Guo, K. M., Wang, H. M., \& Wang, S. 2016, ApJ, 826,6

Zhou, G. P., Zhang, J., \& Wang, J. X. 2016, ApJ, 823, 19 